\title{
NK cells mediate the cumulative analgesic effect of electroacupuncture in a rat model of neuropathic pain
}

Yong-Hui Gao', Jun-Ying Wang ${ }^{1}$, Li-Na Qiao ${ }^{1}$, Shu-Ping Chen ${ }^{1}$, Lian-Hong Tan', Qiu-Ling Xu² and Jun-Ling Liu*

\begin{abstract}
Background: Cumulating evidence has revealed the effectiveness of acupuncture therapy in relieving pain via immunoregulation. However, its underlying mechanism remains unknown. The present study was designed to determine the changes of immunogenic responses at different time-points of electroacupuncture (EA) interventions in neuropathic pain rats.

Methods: The neuropathic pain model was established by ligature of the left sciatic nerve to induce chronic constriction injury (CCI). EA was applied at Zusanli (ST36) and Yanglingquan (GB34) for the EA groups. The thermal pain threshold was detected with an algesia-detector. The subgroups of plasma and splenic lymphocytes were determined via fluorescence-activated cell sorting. Specific inflammatory cytokines were assayed using an ELISA-based bead multiplex assay. The activities of splenic natural killer (NK) cells and cytotoxic T lymphocytes were detected by methyl thiazolyl tetrazolium colorimetric method. For confirming the involvement of NK cell in EA-analgesia, anti-asialo-ganglio-N-tetraosylceramide (anti-asialo-GM1) antibody was given to CCI rats before EA.

Results: Following $\mathrm{CCl}$, the thermal pain threshold of the affected hind footpad was significantly decreased, and increased from the $3^{\text {rd }}$ day to the $12^{\text {th }}$ day after EA interventions, presenting a time-dependent tendency from the 5 th day on. From day 3 to 5 of EA interventions, the percentages and activity of splenic NK cells, concentrations of splenic interleukin-2 (IL-2) and beta-endorphin ( $\beta$-EP) were significantly increased. Meanwhile, the concentrations of plasma $\mathrm{IL}-2, \mathrm{IL}-1 \beta$ and gamma-interferon (IFN- $\gamma$ ) were significantly decreased and returned to the normal level on day 12 following EA. Plasma transforming growth factor- $\beta$ (TGF- $\beta$ ) levels were considerably upregulated on day 5 and 12 following EA. The CD4+/CD8+ T cell ratio was markedly downregulated compared with the control and CCl groups on day 5 and returned to the normal level on day 12 following EA. After depleting NK cells by anti-asialo-GM1 antibody, the increased thermal pain threshold following EA intervention was obviously reduced.
\end{abstract}

Conclusions: Repeated EA interventions have a time-dependent cumulative analgesic effect in neuropathic pain rats, which is closely associated with its regulatory effects on NK cells, splenic IL-2, $\beta-E P$, and plasma IL-2, IL-1 $\beta$, IFN- $\gamma$ and TGF- $\beta$ levels.

Keywords: Acupuncture analgesia, Cumulative effect, Chronic constriction injury, Innate immunity, Acquired immunity, NK cells

\footnotetext{
* Correspondence: 13521898023@163.com

'Department of Physiology, Institute of Acupuncture and Moxibustion, China

Academy of Chinese Medical Sciences, Beijing 100700, China

Full list of author information is available at the end of the article
} 


\section{Background}

Recent data have demonstrated a critical involvement of innate and adaptive immune responses following chronic pain [1,2]. A pivotal event that defines the successful outcome of any inflammatory or injury event is the transition from innate to acquired immunity [3]. The innate immune response is the first line of defense against alarm signals from the injured tissue. The modulation of the innate immunological response to chronic pain has emerged as a promising therapeutic target [4]. T-lymphocyte recruitment plays a role in the establishment of pain chronicity (between days 3-21). In some patient cohorts, antibodies to neuronal antigens have been reported $[5,6]$. Therefore, acquired immunity may also be a good biomarker for the management of clinical neuropathic pain $[4,7]$.

The clinical practice of acupuncture therapy has received worldwide attention as a strategy to attenuate the symptoms of pain [8-10]. Experimental studies have shown that acupuncture intervention effectively relieves chronic neuropathic pain and inflammatory pain in rats $[11,12]$. In the last decade, compelling evidence has demonstrated that the anti-nociceptive effect of electroacupuncture (EA) is closely associated with immunomodulation $[13,14]$. Cumulative evidence revealed that EA intervention could enhances immune function after surgical trauma or neuropathic pain in human [15] and in animals $[16,17]$. However, the mechanism underlying immunoregulation of EA intervention is not clear.

To relieve pain and maintain the efficiency of EA intervention in clinical practice, successive courses of EA treatment are usually necessary for patients with chronic pain. In our previous studies, repeated EA intervention induces a cumulative effect in chronic constriction injury (CCI) rats [18-20]. The analgesic effect of EA peaked after several successive sessions of treatment. This characteristic may be similar to activation of the acquired immune reaction from innate immune response. Therefore, we hypothesized that the cumulative effect of repeated acupuncture intervention may be related to the transition of the innate immune response to the acquired immune response. Thus far, several studies have already examined the effect of EA on innate immune factors. Kim and colleagues [17] demonstrated that the activity of splenic natural killer (NK) cells which are important cellular components of the innate immune response is increased after EA via the induction of cluster of differentiation 94 (CD94). EA also effectively reverses the deleterious immunological changes in the postoperative state by regulating the balance of help $\mathrm{T}$ (Th)1/Th2 cytokines, thereby influencing the acquired immunity [21,22]. However, only few studies have explored the dose-response relationship between the acupuncture stimulation and the change of immunomodulation.

In the present study, we set out to examine the change of acupuncture-associated immunoregulation under neuropathic pain conditions. Specifically, we assessed the role of different lymphocyte subgroups, cytokines, and mediators of analgesia at different time-points of EA interventions to gain insights into its mechanism underlying the conversion from innate to acquired immunity.

\section{Methods}

\section{Animals and grouping}

A total of 50 male Wistar rats (240-300 g) were obtained from the Experimental Animal Center of Peking Union Medical College (Beijing, China), and housed within the animal care facilities in the Institute of Acupuncture and Moxibustion, China Academy of Chinese Medical Sciences. Animals were randomly assigned ( $\mathrm{n}=$ 10 in each group) to sham ligature (CON), $\mathrm{CCI}$ pain model (CCI) and CCI plus three, five, and twelve sessions of EA treatment (EA3d, EA5d, and EA12d, respectively) groups. In order to observe the involvement of NK cells in EA analgesic effect, EA + Anti-asialoganglio-N-tetraosylceramide (anti-asialo-GM1) (EA + anti-asialo-GM1) group and EA + normal rabbit serum $(\mathrm{EA}+$ serum $)$ group were added. All experimental procedures were approved by the Institute of Acupuncture and Moxibustion of China Academy of Chinese Medical Sciences, and performed according to the "Guidelines for Laboratory Animal Care and Use" of the Chinese Ministry of Science and Technology (2006).

\section{$\mathrm{CCl}$ pain model and pain threshold detection}

The CCI model was established by ligating the unilateral sciatic nerve, as previously reported [23]. Briefly, under anesthesia (25\% urethane plus $1.5 \%$ chloralose, $0.4 \mathrm{~mL} /$ $100 \mathrm{~g}$ body weight) and routine sterilization, the left sciatic nerve was exposed at the mid-thigh level by blunt dissection through the biceps femoris. Four constrictive ligatures (4-0 non-absorbable suture) were tied around the nerve at the distal end close to the bifurcation site (about $1 \mathrm{~mm}$ space between every two ligatures). The ligature was all right until a moderate muscular contraction of the leg was seen. The same procedure was performed for rats in the $\mathrm{CON}$ group but without nerve ligature.

The paw withdrawal latency (PWL) (i.e. the thermal pain threshold) of the bilateral hind paws was determined using a 37370 Algesia Detector (Ugo, Italy) 5 days after $\mathrm{CCI}$, and every day thereafter prior to EA treatment. The radiant heat source was focused on the plantar surface of the hindpaw, and light intensity was preset to obtain a baseline latency of approximately $15 \mathrm{sec}$. Each rat underwent two trials with a five-min interval, and the mean value of the two trials was used as the PWL. To minimize differences in individual animals, the difference value of PWL (PWLD) between the healthy and the affected hindpaws was calculated. 


\section{$E A$ intervention}

According to the theory of traditional Chinese medicine, Zusanli (ST36) and Yanglingquan (GB34) are considered to be the most effective acupoints for treating lower back pain, and are commonly used in modern research to study the effects of acupuncture on various physiological regulatory and control systems. ST36 is located $5 \mathrm{~mm}$ beneath the capitulum fibulae and lateralposterior to the knee-joint. GB34 is approximately $5 \mathrm{~mm}$ superior-lateral to ST36. In the present study, 7 days after CCI, animals from the three EA groups were stimulated with EA at bilateral ST36 and GB34. The acupoints were punctured with stainless steel filiform needles (diameter $0.35 \mathrm{~mm}$, length $40 \mathrm{~mm}$, Huatuo; Suzhou Medical Appliance Manufactory, Jiangsu, China) to a depth of approximately $2-3 \mathrm{~mm}$, and stimulated electrically for 30 min using a Han's EA Stimulator (LH202; Neuroscience Research Center, Peking University, Beijing, China). The intensity and frequency of EA were $1 \mathrm{~mA}$ and $15 \mathrm{~Hz}$, respectively. During EA stimulation, the animals were conscious and constrained with a special cloth bag. The treatment was administered once daily continuously for 3, 5 or 12 consecutive days. The other two groups underwent the same procedure but without EA stimulation.

At the end of the EA treatment, all rats from the five groups were deeply anesthetized. Blood samples $(3-5 \mathrm{~mL})$ were obtained through carotid intubation and placed separately in three heparinized tubes. Spleen and hypothalamus tissues were rapidly removed on an ice plate and weighed.

\section{Lymphocyte preparation and fluorescence-activated cell sorting (FACS)}

Spleen tissue was washed with $5 \mathrm{~mL}$ RPMI-1640 medium (Gibco, Grand Island, USA) and then minced with two sterilized glass slides, passed through a $70-\mu \mathrm{m}$ nylon cell strainer, and washed. The suspension was differentially centrifuged over a Percoll gradient (EMB Biosciences, La Jolla, CA) (40\% Percoll, 1200 r/min, $10 \mathrm{~min}, 25^{\circ} \mathrm{C}$ ). The lymphocytes were washed with phosphate-buffered saline (PBS), and then suspended in RPMI-1640 medium, and $10^{6}$ single cell suspensions were prepared.

Whole blood $(100 \mu \mathrm{L})$ and splenic lymphocytes $\left(10^{6}\right)$ were triple-stained with (1) APC-anti-CD3, FITC-antiCD45RA and PE-anti-CD161a, or (2) APC-anti-CD3, PE-anti-CD4 and FITC-anti-CD8a (all from BD Biosciences, San Jose, CA, USA) for $30 \mathrm{~min}$ at $4^{\circ} \mathrm{C}$ in the dark, then washed with PBS and resuspended in FACS staining buffer (BD Biosciences). Three-color analysis was performed on a FACSCalibur (BD Biosciences) with a 100,000-200,000 event count. Data were analyzed using FlowJo software (TreeStar, San Carlos, CA, USA).

\section{Multiplex enzyme-linked immunosorbent assay (ELISA) analysis of plasma and splenic cytokine concentrations}

To prepare splenic aqueous extract for the cytokine concentration assay, the spleen was removed rapidly on an ice plate, and homogenized by a glass tissue homogenizer in an ice cold water bath. The homogenate was then centrifuged $\left(4500 \mathrm{r} / \mathrm{min}\right.$ for $30 \mathrm{~min}$, at $\left.4^{\circ} \mathrm{C}\right)$. The supernatant was collected and stored at $-80^{\circ} \mathrm{C}$ until future use. Plasma was separated by centrifugation $(3000 \mathrm{r} / \mathrm{min}$ for $30 \mathrm{~min}$, at $4^{\circ} \mathrm{C}$ ) and stored at $-80^{\circ} \mathrm{C}$ until use. Concentrations of plasma gamma-interferon (IFN- $\gamma$ ), interleukin-1 beta (IL$1 \beta)$, IL-2, IL-6, IL-4, IL-10, and transforming growth factor beta (TGF- $\beta$ ), and splenic IL-2, IL-12, IL-15 and IFN- $\gamma$ were simultaneously quantified in samples using an ELISA-based bead multiplex assay (Linco Research, St Charles, MO, USA), according to the manufacturer's instruction. Samples were analyzed with a Lumine $\times 100$ plate reader (Linco Research, St Charles, Missouri, USA) to determine the cytokine concentrations. Concentration of each cytokine in the multiplex assay was calculated from calibration curves using individual recombinant proteins as standards. All specimens were tested in duplicate wells to assess inter-assay variability.

\section{NK cell and cytotoxic T lymphocyte (CTL) activities assay}

Splenic $\mathrm{CD}^{+} \mathrm{T}$ cell subset and NK cells were isolated using MagCellect Rat $\mathrm{CD}^{+} \mathrm{T}$ Cell Isolation Kit (R\&D Systems, Minneapolis, MN) and Rat NK Cell Isolation Kit (Yanjin Biotec, Shanghai, China) according to the manufacturer's instructions. The activities of NK cells and CTLs were respectively detected by methyl thiazolyl tetrazolium colorimetric (MTT) method [24]. Briefly, NK cells of each group were collected and seeded in 96 well plates as effect cells at a concentration of $1 \times 10^{5} / \mathrm{ml}$ and cultured $5 \times 10^{5}$ YAC-1 used as target cells. Cocultured NK and YAC-1 cells in 96-well plate at a 50:1 ratio of effector to target (E:T) were simultaneously incubated at $37^{\circ} \mathrm{C}$ in $5 \% \mathrm{CO}_{2}$ for 4 hours in 96 well plates. Then the supernatants were discarded and fresh medium containing $200 \mu \mathrm{L}$ MTT was added to each well and incubated for 4 hours. After the supernatants were discarded, $20 \mu \mathrm{L}$ dimethylsulfoxide was added to each well and mixed thoroughly to dissolve the blue crystals at room temperature. Ten minutes later, when all the crystals dissolved, the plates were read on a plate reader (Bio-Rad, Hercules, CA, USA) at $560 \mathrm{~nm}$. For CTL activity, the effector cells were $2.5 \times 10^{6} / \mathrm{ml}$ splenic $\mathrm{CD}^{+}$ $\mathrm{T}$ lymphocytes and cultured SP2/0 $\left(5 \times 10^{5}\right)$ cells used as target cells. Co-cultured CD8+ T and SP2/0 cells at a $50: 1$ ratio of effector to target $(\mathrm{E}: \mathrm{T})$ were incubated for 24 hours. All MTT assays were performed in triplicate. The activities of CTL and NK cell were calculated using the following formula: NK (or CTL) activity = [1-(OD (E + $\mathrm{T})-\mathrm{ODE}) / \mathrm{ODT}] \times 100 \%$ (ODE, optical density of effect 
cells; ODT, optical density of target cells; OD $(\mathrm{E}+\mathrm{T})$, optical density of co-cultured cells).

\section{Beta-endorphin ( $\beta$-EP) assay}

The hypothalamus tissue samples were placed in tubes containing $1 \mathrm{~mol} / \mathrm{L}(0.5 \mathrm{~mL})$ glacial acetic acid to be homogenized on ice. The tissue lysate was centrifuged (3500 r/min, at $4^{\circ} \mathrm{C}$, for $20 \mathrm{~min}$ ). The concentration of $\beta$-EP was measured by the $\beta$-EP ${ }^{125}$ I radioimmunoassay kit for rat (Sino-UK Institute of Biological Technology, Beijing, China) according to the instruction. All specimens were tested three times to minimize inter-assay variability.

\section{Depletion of NK cells in vivo}

For observing the effect of NK cells on EA analgesia in $\mathrm{CCI}$ rats, other $20 \mathrm{Wistar}$ rats were equally divided into $\mathrm{EA}+$ normal rabbit serum group and EA + Anti-asialoGM1 rabbit antibody group. The procedures of CCI and EA were the same to those mentioned above. The Antiasialo-GM1 rabbit antibody (Abcam Cambridge, UK) was intravenously given at a dose of $80 \mu \mathrm{g}$ on the day before the first EA treatment and once again every 4 days thereafter. Normal rabbit serum was used as control.

\section{Statistical analysis}

All data are presented as the mean \pm standard deviation (SD), and were analyzed by one-way analysis of variance followed by Fisher-Hayter for multiple comparisons with Stata 12.0 software (Stata Corp, College Station, USA). All $P$ values were 2 -sided, and $P<0.05$ was considered significant.

\section{Results}

\section{Effects of successive EA on pain threshold}

No significant differences were found between $\mathrm{CON}$, CCI model and CCI + EA groups in the PWLD before CCI (data not shown). After CCI surgery, rats showed typical neuropathic behavioral responses and a significantly shortened PWL on the nerve ligature side $(P<0.05)$. PWLD was considerably increased in the CCI group compared with the CON group $(P<0.05)$. After three daily sessions of EA, the PWLD was gradually decreased. From the $5^{\text {th }}$ session on, PWLD was significantly lower than that of the CCI group $(P<0.05)$ (Figure 1$)$, thereby presenting a time-dependent effect. It suggests an apparent cumulative analgesic effect of repeated EA intervention.

\section{Effect of different sessions of EA on lymphocytic subpopulations}

Splenic NK cell percentages of the EA5d group, plasma NK cell percentages of the EA3d, EA5d and EA12 d groups were significantly increased compared with the model group $(P<0.05)$. The $\mathrm{CD} 4^{+} / \mathrm{CD}^{+}$ratio of the

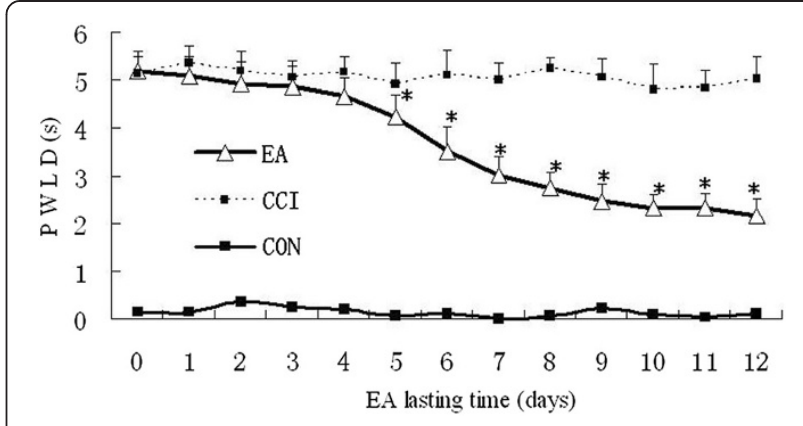

Figure 1 Effect of EA stimulation on PWLD in rats with neuropathic pain ( $n=10$ in each group). PWLD was recorded before and during days 1-12 of EA intervention. Data are presented as the mean $\pm \mathrm{SD}$. ${ }^{*} P<0.05$ vs the $\mathrm{CCl}$ group.

EA5d group was markedly $(P<0.05)$ downregulated compared with the CON and CCI groups (Figure 2).

\section{Effect of repeated EA intervention on splenic NK and CTL cell activities}

The above-mentioned results showed that the percentage of $\mathrm{NK}$ cell was increased and the $\mathrm{CD} 4^{+} / \mathrm{CD} 8^{+}$ratio decreased following EA intervention. CTL is mainly CD8+ T cell and an effector cell in cell immunity. Therefore, we investigated NK and CTL activities to better understand the underlying mechanism of these changes. The results showed that NK cell activity was significantly increased in the EA3d and EA5d groups compared with the CCI group $(P<0.05)$ and returned to the control following 12 sessions of EA. No obvious change in CTL activity was detected in the five groups (Figure 3).

\section{Effect of EA on NK cell activation-associated factors}

To elucidate the mechanism underlying the increase in NK cell activation, NK cell activation-related cytokines were assayed. Splenic IL-2, IL-12 and IL-15 concentrations were slightly but not significantly down-regulated in the CCI group compared with the CON group. The concentration of IFN- $\gamma$ was significantly increased in the $\mathrm{CCI}$ and the EA5d groups $(P<0.05)$. After EA intervention, IL-2 levels of the EA3d and EA5d groups were markedly increased compared with the CCI group $(P<0.05)$. The concentration of splenic IFN- $\gamma$ in the EA12d group was notably reduced to normal level $(P<0.05$, compared with the CCI group) (Figure 4).

$\beta$-EP has also been reported to increase NK cell activity [25]. Therefore, we investigated its concentration in plasma, spleen and hypothalamus. Hypothalamic concentration of $\beta$-EP was dramatically increased after CCI $(P<0.05)$, which did not occur in plasma and spleen. Hypothalamic $\beta$-EP concentration was notably reduced in the EA3d and EA5d groups $(P<0.05)$, while splenic $\beta$-EP levels in EA3d, EA5d, and EA12d groups were considerably upregulated $(P<0.05$, Figure 5$)$. 

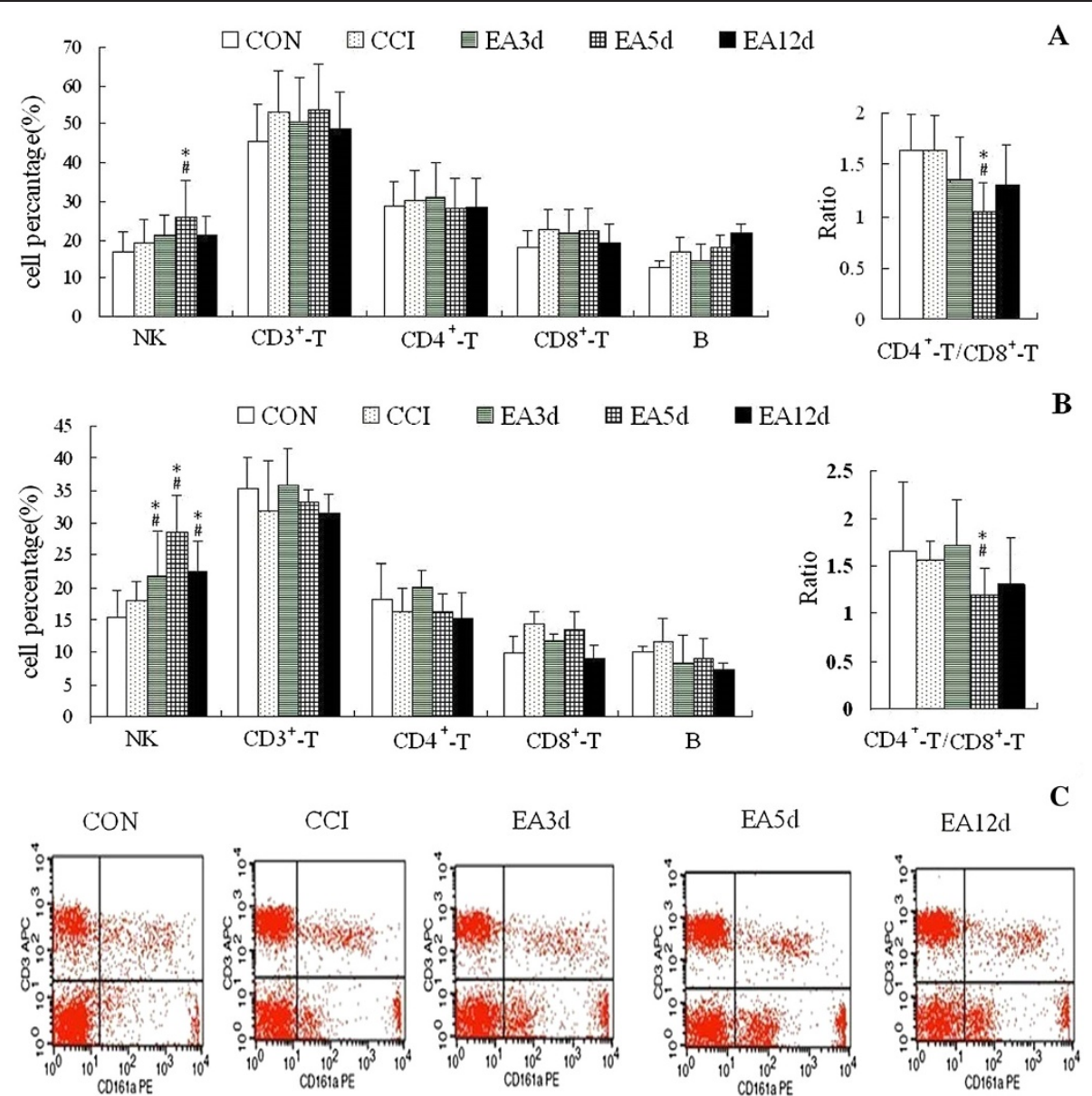

Figure 2 Effect of EA on levels of splenic lymphocytic subgroups (A) and plasma lymphocytic subgroups (B) and a representative FACS scan image of NK cells (C). Data are presented as the mean \pm SD $\left(n=10\right.$ per group). ${ }^{*} P<0.05$ vs the CON group, ${ }^{*} P<0.05$ vs the CCl group.

Effect of EA on plasma pro-inflammatory and anti-inflammatory cytokines

To further elucidate changes of the immune response at different acupuncture intervention time-points, proinflammatory cytokines and anti-inflammatory cytokines in the plasma were examined. In comparison with the control group, plasma pro-inflammatory cytokine concentrations

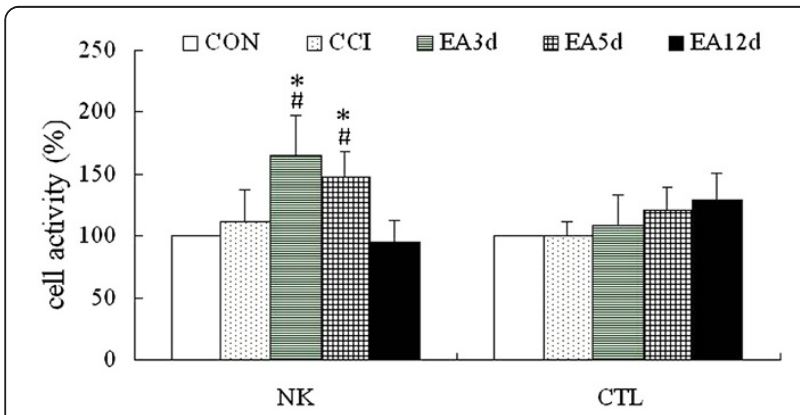

Figure 3 Effect of repeated EA on splenic NK cell and CTL cell activities. Data are presented as the mean \pm SD ( $n=10$ per group). ${ }^{\#} P<0.05$ vs the CON group, ${ }^{*} P<0.05$ vs the CCl group. were apparently (IL- $1 \beta$, IFN- $\gamma, P<0.05$ ) or mildly (IL-2, $P>0.05)$ increased in the CCI group. Anti-inflammatory cytokines, IL-4, IL-10 and TGF- $\beta$ were slightly but not significantly downregulated (Figure 6).

Plasma IL-1 $\beta$ and IFN- $\gamma$ levels of the EA $12 d$ group were markedly downregulated to normal level $(P<0.05)$. In the same group, IL-4 and IL-10 levels were moderately yet not significantly upregulated. TGF- $\beta$ levels in the EA5d and EA12d groups were considerably upregulated $(P<0.05)$, suggesting that repeated EA treatment inhibit inflammation.

Effect of anti-asialo GM1 on pain threshold following EA To confirm the effect of NK cells on EA analgesic, we used anti-asialo GM1 to abolish NK cells in vivo before EA treatment to observe the change of PWLD. As shown in Figure 7, after depleting the NK cell, the decrease of PWLD was not as obvious as the EA group and showed significant difference with EA group from the $6^{\text {th }}$ day on following EA intervention. These results suggest that splenic and plasma NK cells participated in 

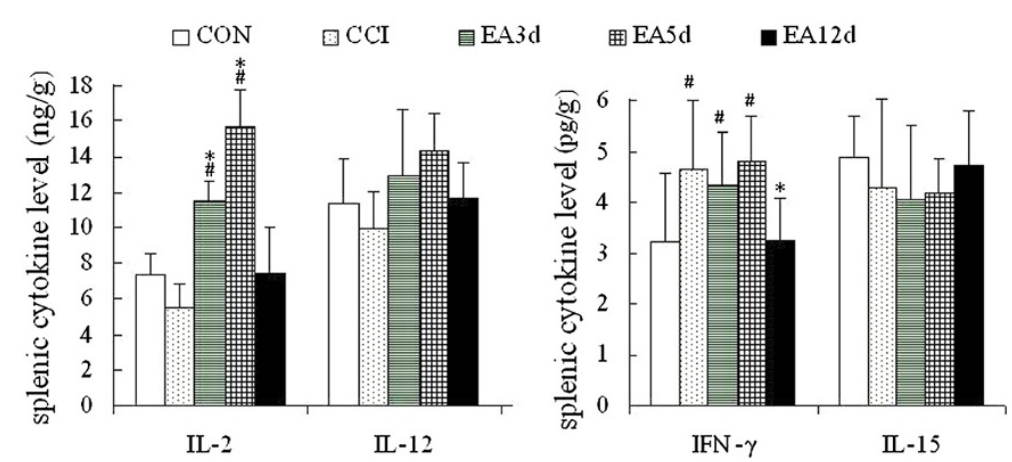

Figure 4 Effect of EA on NK cell activation-associated cytokines. Data are presented as the mean \pm SD ( $n=10$ per group). ${ }^{\#} P<0.05$ vs the CON group, ${ }^{*} P<0.05$ vs the $\mathrm{CCl}$ group.

the repeated EA intervention-induced cumulative analgesic effect in CCI rats.

\section{Discussion}

Although no single theory has successfully offered a unified physiological explanation for the effects of acupuncture, some researchers have proposed that its analgesic action may involve regulation of the body immunity [26]. In the present study, the PWLD was not changed at the early stage of EA (days 1-3), but increased considerably after 4-5 sessions of EA intervention and presented a time-dependent manner. This result demonstrated that the body's response to EA at the early stage may be different from that at the "cumulative" stage. Under CCI, the "cumulative" effect commenced from 4-5 days following EA. Interestingly, this point is also the shifting time from innate immunity to acquired immunity. Therefore, we selected the time points of day 3 and 5 following EA to examine the change of immune regulation from lymphocyte subgroups and related cytokines.

We observed that lymphocyte subpopulation NK cell was increased both in their activity and in quantity in the spleen and peripheral blood following EA intervention. Next, we examined changes of the NK cell activationassociated factors following acupuncture treatment to elucidate the mechanism of the increase in NK cells. IL-2 and $\beta$-EP, two efficacious activators of NK cells, were increased. Some studies have shown that the effect of $\beta$-EP on NK cell activity is dependent on its location [27]. Our results demonstrated that in the hypothalamus, the $\beta$-EP mainly functions as an analgesic substance. However, in the spleen, its role is complex because of a possible combination with IL-2 to enhance NK cell activity. These results confirmed that NK cell may be an effector at the early stage of EA to initiate its cumulative effect, which was evidenced by the studies of $\mathrm{Yu}[28,29]$.

It has been demonstrated that loss of larger myelinated fibers in a CCI model causes changes in the neurogenic component, which induced a recruitment of immune cells at the injury site of the peripheral nerve. Thus, changes in the profile of neurochemicals involving neuropathic pain affect the number and activity of immune cells in the plasma or spleen [30]. Trauma, surgical stress and chronic pain are often associated with immune suppression and increased susceptibility to infection [21]. Sunagawa' study showed that splenic NK cell activity was suppressed by ligation of unilateral mental nerve in rats [31]. NK cells are the third subset of lymphocytes and are important for the early immune response against infections. It thus appears to be important to prevent the relative suppression
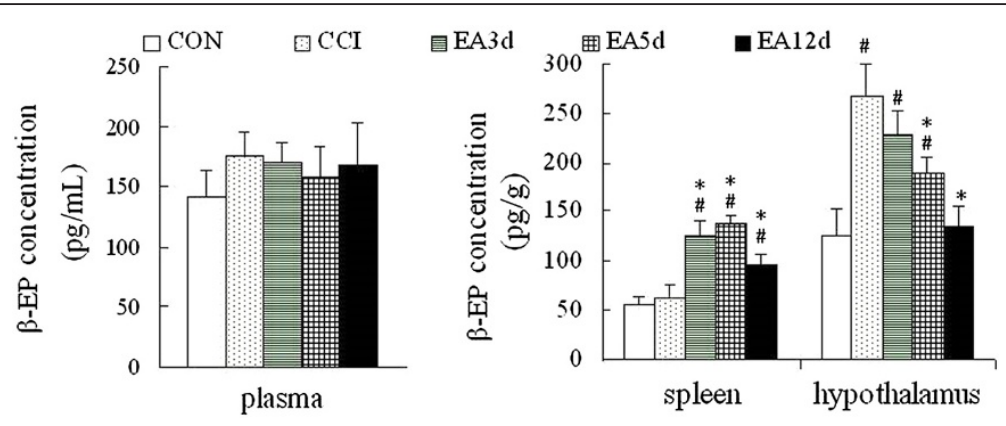

Figure 5 Effect of EA on $\beta$-EP concentration. Data are presented as the mean \pm SD $\left(n=10\right.$ per group). ${ }^{\sharp} P<0.05$ vs the CON group, ${ }^{*} P<0.05$ vs the $\mathrm{CCl}$ group. 


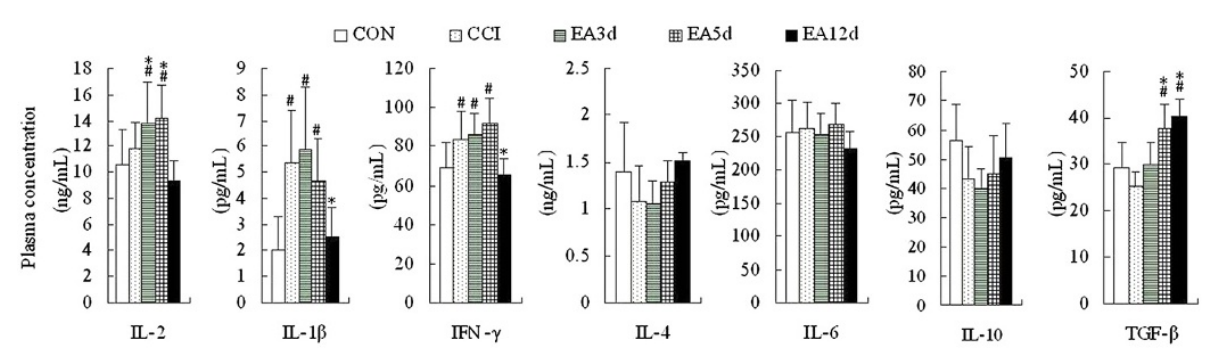

Figure 6 Effect of EA on plasma cytokine concentrations in CCI rats. Data are presented as the mean $\pm \mathrm{SD}\left(\mathrm{n}=10\right.$ per group). ${ }^{\#} P<0.05 \mathrm{vS}$ the CON group, ${ }^{*} P<0.05$ vs the $C C l$ group.

of NK cell to maintain immune function. Our study suggests that EA stimulation has a positive role in the immunoregulation after CCI through increasing the NK cell function. We also observed that the ratio of CD4+/CD8+ $\mathrm{T}$ cell was decreased following five sessions of EA. This may suggest a change in the levels of some T-lymphocyte subgroups. However, we did not find the exact source of this change because of the wide varieties of the subgroups. $\mathrm{T}$ lymphocyte may have a role in the maintenance of neuropathic pain because T-lymphocyte deficient mice were found to have a reduction in injury-induced hypersentivity but adoptive transfer reversed this effect [32]. At the same time, T lymphocyte that infiltrated into the central nervous system interacted with microglia and helped maintain neuropathic pain by the secretion of proinflammatory mediators and also possibly by facilitating the activation of astrocytes [33]. Moreover, some studies also suggest that modulation of T- lymphocyte phenotype

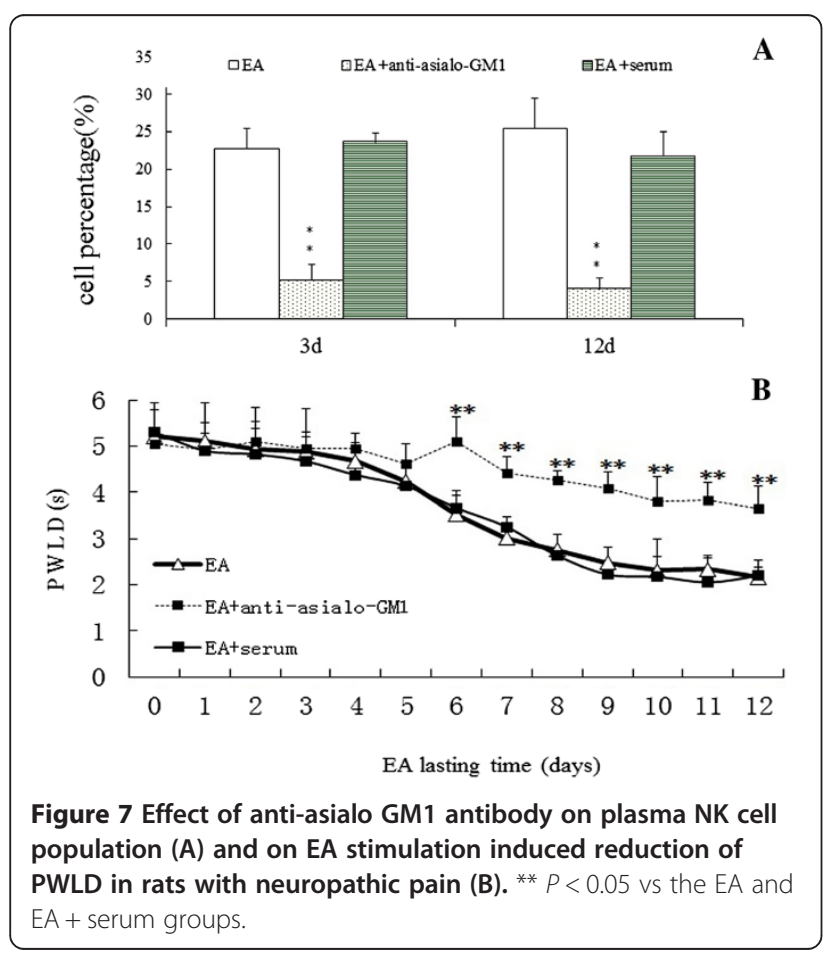

might be one approach to the treatment of neuropathic pain [1]. Therefore, further experiments to explore the exact changes in $\mathrm{T}$ cell subgroups (Th cell, Tc cell, Th17, Treg cells...) following CCI and EA should be performed.

Cytokines have emerged as essential regulators of lymphocyte trafficking, which is necessary to turn an innate immune response into an adaptive response [34,35]. IFN- $\gamma$, IL- 2 and TGF- $\beta$ are all cytokines that support cellular immunity. After three sessions of EA intervention, and particularly following five successive sessions, the concentrations of IL- 2 and TGF- $\beta$ in the spleen or plasma were increased, suggesting an activation of adaptive immunity. Interestingly, NK cells can furthermore function as "adaptive effectors" once activated by T cellinduced IFN- $\gamma$ or when IgG eventually elicits antibodydependent cell cytotoxicity [36-38]. Therefore, NK cells not only synergize with innate immunity against danger signals but also shape the adaptive immune system [39], thus highlighting its importance as an important adjuvant during the development of immunoregulation after acupuncture intervention. NK cells are also an important source of IFN- $\gamma$ which induces the production of chemokines (Interferon-inducible Protein 10, IL-8, etc.) downstream of toll-like receptor (TLR) activation from resident tissue cells [40]. These chemokines can attract antigenspecific $\mathrm{CD}^{+}$or $\mathrm{CD}^{+} \mathrm{T}$ lymphocytes into the injured tissue to establish organ-specific immunity, which constitutes an important link between the innate and adaptive immune responses. This link is essential for protective immunity. However, the type of cellular immunity that occurred in the present study remains elusive because we did not examine the subpopulations of B cells and subpopulations of Th and CTL cells, which eventually elicit an "elimination" effect on danger signals. In the present study, CCI increased plasma concentrations of the proinflammatory cytokines, IFN- $\gamma$, IL- $1 \beta$, and IL- 2 . However, no notable changes were seen in the levels of the antiinflammatory cytokines, IL-4, IL- 6 and IL-10. These results also suggested an imbalance of TH1/TH2. Following 12 sessions of EA, plasma levels of IL-1 $\beta$ and IFN- $\gamma$ were significantly decreased, and the anti-inflammatory TGF- $\beta$ was considerably increased, implying that after undergoing 
approximately 10 days of cellular immunity, the balance between $\mathrm{TH} 1$ and $\mathrm{TH} 2$ was restored.

The innate and adaptive immune systems are clearly two interdependent parts of a single integrated immune system. The innate immune response not only provides the first line of defense against "danger", but also provides the biological context of the "danger signal" that determines which antigens the acquired immune system responds to and the nature of that response to instruct the adaptive immune system to mount a response. In the present study, the most notable decrease in PWLD occurred between day 3 and 5 of EA interventions. This time point was consistent with that of the innate immunity to motivate the acquired immunity. Moreover, following five sessions of EA, the ratio of CD4 + T/CD8+ T cell was decreased, which may suggest a change in the levels of some subgroups of CD4+ or a downregulation of subgroups of CD8+, or a combination of the two. These cells are acquired immunity response cells. These coincidences may suggest that the different stage of EA interventions was related to the shift from the innate to the acquired immune responses. However, more sophisticated researches are definitely necessary for supporting this hypothesis.

\section{Conclusions}

Successive EA interventions have a time-dependent cumulative analgesic effect in neuropathic pain rats, which is closely associated with its regulatory effects on NK cells, splenic IL-2, $\beta$-EP, and plasma IL-2, IL-1 $\beta$, IFN- $\gamma$ and TGF$\beta$ levels. As an effect cell involving this cumulative analgesic effect, NK cell may be a functional bridge between the cumulative analgesic effect of EA and the immune activity and may be a target for EA analgesia. Results of the present study provide the first insight into the origins of innate to adaptive immunity following EA interventions in a rat model of neuropathic pain.

\section{Abbreviations}

CCI: Chronic constrictive injury; EA: Electroacupuncture; CON: Normal control; PWL: Paw withdrawal latency; PWLD: Difference value of PWL; NK: Natural killer; $\beta$-EP: Beta-endorphin; CD: Cluster of differentiation; Th: Help T cell; FACS: Fluorescence-activated cell sorting; IFN-Y: Gamma-interferon; IL: Interleukin; TGF- $\beta$ : Transforming growth factor beta; ELISA: Enzyme-linked immunosorbent assay; CTL: Cytotoxic T lymphocyte; MTT: Methyl thiazolyl tetrazolium.

\section{Competing interests}

The authors declared that they have no competing interests.

\section{Authors' contributions \\ YHG and JLL conceived this study and wrote the manuscript. YHG, JYW, LNQ, SPC and LHT carried out the experiment. LHT and QLX executed statistical analysis. All authors read and approved the final manuscript.}

\section{Acknowledgments}

This study was jointly supported by the National Nature Science Foundation $(81102647,90709031,81273830)$ and the Special Project of Chinese Medicine (973) of the National Basic Research Program of China (2007CB512505, 2013CB531904).

\section{Author details}

'Department of Physiology, Institute of Acupuncture and Moxibustion, China Academy of Chinese Medical Sciences, Beijing 100700, China. ${ }^{2}$ Hainan Medical University, Haikou, Hainan Province 571101, China.

Received: 20 December 2013 Accepted: 13 August 2014 Published: 26 August 2014

\section{References}

1. Calvo M, Dawes JM, Bennett DL: The role of the immune system in the generation of neuropathic pain. Lancet Neurol 2012, 11(7):629-642.

2. Ren $K$, Dubner R: Interactions between the immune and nervous systems in pain. Nat Med 2010, 16(11):1267-1276.

3. Jones SA: Directing transition from innate to acquired immunity: defining a role for IL-6. J Immunol 2005, 175(6):3463-3468.

4. Guo LH, Schluesener HJ: The innate immunity of the central nervous system in chronic pain: the role of Toll-like receptors. Cell Mol Life Sci 2007, 64(9):1128-1136.

5. Vincent $A$, Bien CG, Irani SR, Waters P: Autoantibodies associated with diseases of the CNS: new developments and future challenges. Lancet Neurol 2011, 10(8):759-772.

6. Kohr D, Singh P, Tschernatsch M, Kaps M, Pouokam E, Diener M, Kummer W, Birklein F, Vincent A, Goebel A, Wallukat G, Blaes F: Autoimmunity against the beta2 adrenergic receptor and muscarinic-2 receptor in complex regional pain syndrome. Pain 2011, 152(12):2690-2700.

7. Austin PJ, Moalem-Taylor G: The neuro-immune balance in neuropathic pain: involvement of inflammatory immune cells, immune-like glial cells and cytokines. J Neuroimmunol 2010, 229(1-2):26-50.

8. Leibing E, Leonhardt U, Koster G, Goerlitz A, Rosenfeldt JA, Hilgers R, Ramadori G: Acupuncture treatment of chronic low-back pain - a randomized, blinded, placebo-controlled trial with 9-month follow-up. Pain 2002, 96(1-2):189-196.

9. Carlsson CP, Sjolund BH: Acupuncture for chronic low back pain: a randomized placebo-controlled study with long-term follow-up. Clin $J$ Pain 2001, 17(4):296-305

10. Shen YF, Goddard G: The short-term effects of acupuncture on myofascial pain patients after clenching. Pain Pract 2007, 7(3):256-264.

11. Kim HN, Park JH, Kim SK, Sun B, Koo S, Choi SM, Bae H, Min Bl: Electroacupuncture potentiates the antiallodynic effect of intrathecal neostigmine in a rat model of neuropathic pain. J Physiol Sci 2008, 58(5):357-360.

12. Yim YK, Lee H, Hong KE, Kim YI, Lee BR, Son CG, Kim JE: Electroacupuncture at acupoint ST36 reduces inflammation and regulates immune activity in collagen-induced arthritic mice. Evid Based Complement Alternat Med 2007, 4(1):51-57.

13. Quispe-Cabanillas JG, Damasceno A, von Glehn F, Brandao CO, Damasceno BP, Silveira WD, Santos LM: Impact of electroacupuncture on quality of life for patients with relapsing-remitting multiple sclerosis under treatment with immunomodulators: a randomized study. BMC Complement Altern Med 2012, 12:209.

14. Lin JG, Chen WL: Acupuncture analgesia: a review of its mechanisms of actions. Am J Chin Med 2008, 36(4):635-645.

15. Cabyoglu MT, Ergene N, Tan U: The mechanism of acupuncture and clinical applications. Int J Neurosci 2006, 116(2):115-125.

16. Wang J, Zhao H, Mao-Ying QL, Cao XD, Wang YQ, Wu GC: Electroacupuncture downregulates TLR2/4 and pro-inflammatory cytokine expression after surgical trauma stress without adrenal glands involvement. Brain Res Bull 2009, 80(1-2):89-94.

17. Kim CK, Choi GS, Oh SD, Han JB, Kim SK, Ahn HJ, Bae H, Min Bl: Electroacupuncture up-regulates natural killer cell activity identification of genes altering their expressions in electroacupuncture induced up-regulation of natural killer cell activity. J Neuroimmunol 2005, 168(1-2):144-153.

18. Gao Y, Chen S, Xu Q, Yu K, Wang J, Qiao L, Meng F, Liu J: Proteomic analysis of differential proteins related to anti-nociceptive effect of electroacupuncture in the hypothalamus following neuropathic pain in rats. Neurochem Res 2013, 38(7):1467-1478.

19. Liu JL, Chen SP, Gao YH, Meng FY, Wang SB, Wang JY: Effects of repeated electroacupuncture on beta-endorphin and adrencorticotropic hormone levels in the hypothalamus and pituitary in rats with chronic pain and ovariectomy. Chin J Integr Med 2010, 16(4):315-323. 
20. Gao YH, Chen SP, Wang JY, Qiao LN, Meng FY, Xu QL, Liu JL: Differential proteomics analysis of the analgesic effect of electroacupuncture intervention in the hippocampus following neuropathic pain in rats. BMC Complement Altern Med 2012, 12:241.

21. Wang K, Wu H, Wang G, Li M, Zhang Z, Gu G: The effects of electroacupuncture on $\mathrm{TH} 1 / \mathrm{TH} 2$ cytokine mRNA expression and mitogen-activated protein kinase signaling pathways in the splenic $T$ cells of traumatized rats. Anesth Analg 2009, 109(5):1666-1673.

22. Carneiro ER, Xavier RA, De Castro MA, Do Nascimento CM, Silveira VL: Electroacupuncture promotes a decrease in inflammatory response associated with Th1/Th2 cytokines, nitric oxide and leukotriene B4 modulation in experimental asthma. Cytokine 2010, 50(3):335-340.

23. Bennett GJ, Xie YK: A peripheral mononeuropathy in rat that produces disorders of pain sensation like those seen in man. Pain 1988, 33(1):87-107.

24. Dou J, Chen JS, Wang J, Chen GB, Zhao FS, Tang Q, Fang XS, Chu LL, Pan M: Novel constructs of tuberculosis gene vaccine and its immune effect on mice. Cell Mol Immunol 2005, 2(1):57-62.

25. Chen CP, Boyadjieva NI, Advis JP, Sarkar DK: Ethanol suppression of the hypothalamic proopiomelanocortin level and the splenic NK cell cytolytic activity is associated with a reduction in the expression of proinflammatory cytokines but not anti-inflammatory cytokines in neuroendocrine and immune cells. Alcohol Clin Exp Res 2006, 30(11):1925-1932.

26. Kavoussi B, Ross BE: The neuroimmune basis of anti-inflammatory acupuncture. Integr Cancer Ther 2007, 6(3):251-257.

27. Panerai $A E$, Sacerdote P: Beta-endorphin in the immune system: a role at last? Immunol Today 1997, 18(7):317-319.

28. Yu Y, Kasahara T, Sato T, Asano K, Yu G, Fang J, Guo S, Sahara M, Hisamitsu T: Role of endogenous interferon-gamma on the enhancement of splenic NK cell activity by electroacupuncture stimulation in mice. J Neuroimmunol 1998, 90(2):176-186.

29. Yu Y, Kasahara T, Sato T, Guo SY, Liu Y, Asano K, Hisamitsu T: Enhancement of splenic interferon-gamma, interleukin-2, and NK cytotoxicity by S36 acupoint acupuncture in F344 rats. Jpn J Physiol 1997, 47(2):173-178.

30. Herzberg U1, Murtaugh M, Beitz AJ: Chronic pain and immunity: mononeuropathy alters immune responses in rats. Pain 1994, 59(2):219-225.

31. Sunagawa M1, Okada M, Guo SY, Hisamitsu T: Splenic natural killer cell activity is suppressed by ligation of unilateral mental nerve in rats. Masui 2000, 49(3):250-254.

32. Cao L1, DeLeo JA: CNS-infiltrating CD4+ T lymphocytes contribute to murine spinal nerve transection-induced neuropathic pain. Eur I Immunol 2008, 38(2):448-458

33. Grace PM1, Rolan PE, Hutchinson MR: Peripheral immune contributions to the maintenance of central glial activation underlying neuropathic pain. Brain Behav Immun 2011, 25(7):1322-1332.

34. Luster AD: The role of chemokines in linking innate and adaptive immunity. Curr Opin Immunol 2002, 14(1):129-135.

35. Young HA, Ortaldo J: Cytokines as critical co-stimulatory molecules in modulating the immune response of natural killer cells. Cell Res 2006, 16(1):20-24.

36. Caligiuri MA: Human natural killer cells. Blood 2008, 112(3):461-469.

37. Terrazzano $G$, Carbone E: NK cells blur the frontier between innate and acquired immunity. Front Immunol 2013, 3:400.

38. Vivier $E$, Tomasello E, Baratin M, Walzer T, Ugolini S: Functions of natural killer cells. Nat Immunol 2008, 9(5):503-510.

39. Brilot F, Strowig T, Munz C: NK cells interactions with dendritic cells shape innate and adaptive immunity. Front Biosci 2008, 13:6443-6454.

40. Khan IA, MacLean JA, Lee FS, Casciotti L, DeHaan E, Schwartzman JD, Luster AD: IP-10 is critical for effector T cell trafficking and host survival in Toxoplasma gondii infection. Immunity 2000, 12(5):483-494.

doi:10.1186/1472-6882-14-316

Cite this article as: Gao et al:: NK cells mediate the cumulative analgesic effect of electroacupuncture in a rat model of neuropathic pain. BMC Complementary and Alternative Medicine 2014 14:316.

\section{Submit your next manuscript to BioMed Central and take full advantage of:}

- Convenient online submission

- Thorough peer review

- No space constraints or color figure charges

- Immediate publication on acceptance

- Inclusion in PubMed, CAS, Scopus and Google Scholar

- Research which is freely available for redistribution

Submit your manuscript at www.biomedcentral.com/submit
C Biomed Central 